\title{
Time Modulation of K-electron Capture Decay of Hydrogen-Like Ions with Multiphoton Resonance Transitions
}

\author{
I. M. Pavlichenkov ${ }^{1, *}$ \\ ${ }^{1}$ Russian Research Center "Kurchatov Institute", Moscow, 123182, Russia.
}

(Dated: October 30, 2018)

\begin{abstract}
The multiphoton resonance transitions between ground hyperfine states are used for the time modulation of the electron capture decay of hydrogen like ions with the Gamow-Teller transition $1^{+} \rightarrow 0^{+}$. The proposed mechanism offers a time oscillating decay with the frequency up to 0.1 Hz. The experiment to observe the modulation is proposed for ions stored in a Penning trap. An attempt to understand the GSI anomaly with multiple photon transitions is made.

PACS numbers: 23.40.-s, 32.30.Dx, 32.80.Rm
\end{abstract}

Longstanding attempts have been made to change artificially the decay of radioactive nuclei using different external factors. It was suggested by Segré [1] that the orbital electron capture (EC) decay rates depend on the density of atomic electrons within a nucleus. Thus, environmental conditions may alter electron densities and affect EC decay rates. However this effect is small and does not exceed the fractions of percentage point.

The introduction of Penning traps and storage rings into nuclear physics has allowed to study radioactive hydrogen-like (H-like) ions. Recently the single ion spectroscopy technique with time resolution less than $1 \mathrm{~s}$ has been developed at GSI Darmstadt. With this elegant method, the oscillatory modulation of the exponential EC decay for the ${ }^{140} \mathrm{Pr}^{58+}$ and ${ }^{142} \mathrm{Pm}^{60+}$ ions was found in [2]. The modulation period of $7.1 \mathrm{~s}$ is incommensurable with all the energy intervals involved in the experiment with exclusion of the neutrino mass difference. Perhaps this is the main motivation of the Letter [3] which relates the anomaly to neutrino mixing.

Our motivation for this work is to show that there is a mechanism related to Rabi oscillations which offers such a small period. To observe the time modulation of the EC decay, we propose to apply Penning trap mass spectrometry with ions in the intermediate mass region having the "sterile" relative to the EC decay hyperfine state. We analyze also the effect of the magnetic fields of dipole and quadrupole magnets in the Experimental Storage Ring (ESR) on the GSI oscillations.

The $1 s_{1 / 2}$ state of a H-like ion has two hyperfine levels with the total angular momentum $F=I \pm 1 / 2$, where $I$ is the nuclear spin. In the case of a positive nuclear magnetic moment $\mu$, the ground state of an ion has $F=$ $I-1 / 2$, whereas for negative $\mu$, the order of these energy levels is reversed. When $I=1$, the hyperfine splitting of the $1 s_{1 / 2}$ state is given by [4]

$$
\Delta E=\frac{2 \alpha(\alpha Z)^{3}\left(\mu / \mu_{N}\right) m^{2} c}{M\left(2 \sqrt{1-(\alpha Z)^{2}}-1\right) \sqrt{1-(\alpha Z)^{2}}}
$$

\footnotetext{
*Electronic address: pavi@mbslab.kiae.ru
}

where $m, M, \mu_{N}$, and $\alpha$ are the masses of electron and proton, nuclear magneton, and the fine-structure constant, respectively. For nuclei with $I^{\pi}=1^{+}$, the EC decay from the $F=3 / 2$ state is forbidden because fully ionized daughter nuclei have $I^{\pi}=0^{+}$. This feature of such ions may be used for observing the time modulation of the EC decay.

The oscillatory behavior of the EC decay of such ions can be obtained with the Rabi resonance method used in nuclear magnetic spectroscopy. We consider an ion in a static magnetic field in the $z$ direction, resulting in the Zeeman splitting of both hyperfine levels. Transitions between the Zeeman components of these two states are driven by the transverse oscillating field along the $x$ direction with frequency $\omega$. When the frequency $\omega$ is tuned to $\Delta E / \hbar$, the oscillation of populations for the states $F=1 / 2, M$ and $F=3 / 2,-M$, where $M=1 / 2$ or $-1 / 2$, is modulated with the Rabi frequency, which is proportional to the amplitude of the driving field. Because the EC decay probability is proportional to the occupation of the $F=1 / 2$ state, the Rabi frequency may be observed as the time modulation of the EC decay rates. The time modulation of EC-decay is also possible with the resonance transitions involving several field quanta.

Penning trap.- A single H-like ion in a Penning trap is an ideal object for observing the modulation of the EC decay. The ion having a charge-to-mass ratio $q / M$ is confined in a strong magnetic field $\mathbf{B}\left(0,0, B_{0}\right)$ superimposed with a weak electrostatic quadrupole field [5]. The solution of the motion equations yields the cyclotron, axial and magnetron harmonic oscillations. The ion mass is determined via the determination of the free-space cyclotron frequency $\omega_{c}=q B_{z} / M c$ which derived from three measured harmonic frequencies. The single ion spectroscopy developed in the ESR experiments can be installed at a Penning trap [6]. In this technique, it is possible to realize the fully controllable experiment on the time modulation of the EC decay by single- and multiphoton resonance.

We are interested in the time evolution of the two hyperfine states of an ion exposed by the trap magnetic field $\mathbf{B}$ and irradiated by the in-plane elliptically polarized driving field $\mathbf{b}\left(b_{x} \sin \omega t, 0, b_{z} \cos \omega t\right)$. The strength 
of the latter is restricted by the frequency resolution of the single ion spectroscopy. It is necessary for a shift in the cyclotron frequency $\omega_{c}$ caused by the influence of the field $\mathbf{b}$ to be small compared to the change in $\omega_{c}$ due to the transition from a parent ion to a daughter nucleus. As a rough approximation, this gives $b<B_{0} Q_{E C} /\left(M c^{2}\right)$, where $Q_{E C}$ is the decay energy.

The total Hamiltonian is $H=H_{0}+U(t)+V(t)$, where $H_{0}=H_{D}+(e / 2)[\boldsymbol{\alpha} \mathbf{r}] \cdot \mathbf{B}$ describes the $1 s_{1 / 2}$ state of a H-like ion in the Penning trap. Here $H_{D}$ is the Dirac Hamiltonian with hyperfine interaction and $\boldsymbol{\alpha}$ is the Dirac matrix. The eigenfunctions and eigenvalues of $H_{0}$ are $|+\rangle=\left|3 / 2, M_{+}\right\rangle, E_{3 / 2}+(1 / 3) g \mu_{B} B_{0} M_{+}=\hbar \omega_{3}$ and $|-\rangle=|1 / 2, M\rangle, E_{1 / 2}-(1 / 3) g \mu_{B} B_{0} M=\hbar \omega_{1}$. Here $\mu_{B}$ is the Bohr magneton and $g$ is the gyromagnetic ratio of the bound electron in a $\mathrm{H}$-like ion. The terms $U(t)=(e / 2)[\boldsymbol{\alpha} \mathbf{r}]_{z} b_{z} \cos \omega t$ and $V(t)=(e / 2)[\boldsymbol{\alpha} \mathbf{r}]_{x} b_{x} \sin \omega t$ represent the diagonal and off-diagonal parts of the interaction between an ion and the driving field.

There are three independent transitions between the lower and upper Zeeman sub-states which can be used for the modulation of the EC decay:

(i) The transitions between the states $|3 / 2,+1 / 2\rangle$ and $|1 / 2,-1 / 2\rangle$ or $|3 / 2,-1 / 2\rangle$ and $|1 / 2,+1 / 2\rangle$ with the energy $\Delta E=E_{3 / 2}-E_{1 / 2}=\hbar \omega_{0}$ regardless of the sign of $\mu$.

(ii) The transitions between the states $|3 / 2, \mp 3 / 2\rangle$ and $|1 / 2, \mp 1 / 2\rangle$ for $\mu>0$ (upper sign) and $\mu<0$ (lower sign) with the energy $\Delta E-(2 / 3) g \mu_{B} B_{0}$.

(iii) The transitions between the states $|3 / 2, \pm 3 / 2\rangle$ and $|1 / 2, \pm 1 / 2\rangle$ for $\mu>0$ (upper sign) and $\mu<0$ (lower sign) with the energy $\Delta E+(2 / 3) g \mu_{B} B_{0}$.

Thus, we have the two discrete states $|+\rangle$ and $|-\rangle$ with the energies $\epsilon_{3}=\hbar \omega_{3}$ and $\epsilon_{1}=\hbar\left(\omega_{1}-i \lambda / 2\right)$, where $\lambda$ is the EC decay constant. Let the amplitudes for an ion to be in these states are $a_{+}(t)$ and $a_{-}(t)$. Then the system evolves according to the equations

$$
\begin{aligned}
& i \hbar \dot{a}_{-}=\left(\epsilon_{1}+U_{-} \cos \omega t\right) a_{-}+V_{-+} a_{+} \sin \omega t, \\
& i \hbar \dot{a}_{+}=\left(\epsilon_{3}+U_{+} \cos \omega t\right) a_{+}+V_{+-} a_{-} \sin \omega t,
\end{aligned}
$$

where $U_{+}=g \mu_{B} b_{z} M_{+} / 3, U_{-}=-g \mu_{B} b_{z} M_{-} / 3$,

$$
V_{+-}=\left\{\begin{array}{l}
\hbar V=\frac{1}{3 \sqrt{ } 2} g \mu_{B} b_{x} \quad \text { for (i), } \\
\hbar \tilde{V}=-\frac{M_{+}}{\sqrt{ } 3 \mid M_{+}} g \mu_{B} b_{x} \quad \text { for (ii) and (iii). }
\end{array}\right.
$$

To eliminate the diagonal oscillating terms in (2), we perform the transformation

$$
a_{ \pm}=A_{ \pm} \exp \left[-i\left(\omega_{1}+\omega_{3}\right) t / 2-i\left(U_{ \pm} / \hbar \omega\right) \sin \omega t\right],
$$

which reduces the equations for the transitions (i) to the form

$$
\begin{aligned}
& i \dot{A}_{-}=-\frac{1}{2}\left(\omega_{0}+i \lambda\right) A_{-}+V A_{+} \sin \omega t, \\
& i \dot{A}_{+}=\frac{1}{2} \omega_{0} A_{+}+V A_{-} \sin \omega t,
\end{aligned}
$$

The two other transitions are described by the equations

$$
\begin{aligned}
& i \dot{A}_{-}=-\frac{1}{2}\left(\tilde{\omega}_{0}+i \lambda\right) A_{-}+\tilde{V} A_{+} \exp \left(i \frac{U}{\omega} \sin \omega t\right) \sin \omega t \\
& i \dot{A}_{+}=\frac{1}{2} \tilde{\omega}_{0} A_{+}+\tilde{V} A_{-} \exp \left(-i \frac{U}{\omega} \sin \omega t\right) \sin \omega t
\end{aligned}
$$

where $\tilde{\omega}_{0}=\omega_{3}-\omega_{1}, U=\left(U_{-}-U_{+}\right) / \hbar$. Through the use of the expansion of an exponent in the Bessel functions, these equations are converted to

$$
\begin{aligned}
& i \dot{A}_{-}=-\frac{1}{2}\left(\tilde{\omega}_{0}+i \lambda\right) A_{-}-i \tilde{V} A_{+} \sum_{m=-\infty}^{\infty} J_{m}^{\prime}\left(\frac{U}{\omega}\right) e^{i m \omega t} \\
& i \dot{A}_{+}=\frac{1}{2} \tilde{\omega}_{0} A_{+}+i \tilde{V} A_{-} \sum_{m=-\infty}^{\infty} J_{m}^{\prime}\left(\frac{U}{\omega}\right) e^{-i m \omega t}
\end{aligned}
$$

where $J_{m}^{\prime}$ is the derivative of the Bessel function. The equations (5) and (7) describe an ion with a positive nuclear magnetic moment $\mu$. When $\mu<0$, it is necessary to change the sign of $\omega_{0}$ and $\tilde{\omega}_{0}$. Up to this point all of the equations are exact.

The equations (5) for $\lambda=0$ are the basic ones in the theory of the resonance interaction of the two level system with a periodic field. When $\omega \approx \omega_{0}$, we get the usual Rabi formula for amplitudes replacing the sine by one exponent [7]. For the K-photon resonance when $\omega_{0} \approx K \omega$, Shirley [8] found the approximate solution for a weak driving field. In the adiabatic approximation, $K \gg 1$, the solution of (5) was first obtained by Zaretskii and Krainov [9] and then by Duvall et al. [10]. In the weak field regime, $b_{x} \ll B_{0}$, which is adequate for the Penning trap experiment, we use Sambe's perturbation approach 11] provided that $\lambda \ll \omega$. In the $K$-th order of the perturbation theory in the quasienergy representation, Eqs. (5) can be approximated by

$$
\begin{aligned}
& i \dot{A}_{-}=-\frac{1}{2}\left(\omega_{\text {res }}-K \omega+i \lambda\right) A_{-}+\frac{1}{2} \Gamma_{K} A_{+}, \\
& i \dot{A}_{+}=\frac{1}{2}\left(\omega_{\text {res }}-K \omega\right) A_{+}+\frac{1}{2} \Gamma_{K} A_{-},
\end{aligned}
$$

where

$$
\omega_{r e s}=\omega_{0}\left[1+\frac{K^{2}}{K^{2}-1}\left(\frac{g \mu_{B} b_{x}}{3 \sqrt{2} \hbar \omega_{0}}\right)^{2}\right], \quad K>1
$$

is the shift of the resonance frequency related to the dynamic Zeeman effect which is referred as the BlochSiegert shift. The shift is of the order of $\omega_{0}\left(b_{x} / B_{0}\right)$. For $K=1$ the coefficient before round brackets is equal to $1 / 4$. The half width of $K$-photon resonance is

$$
\Gamma_{K}=\frac{2 \omega}{[(K-1) ! !]^{2}}\left(\frac{g \mu_{B} b_{x}}{6 \sqrt{2} \hbar \omega}\right)^{K}
$$

the photon number $K$ being odd for these transitions.

We now turn to a close examination of Eqs. (7) for the transitions (ii) and (iii). When the frequency $K \omega$ ( $K$ is even or odd integer) is nearly resonant with the energy separation $\tilde{\omega}_{0}$ of the two states, the term with $m=K$ represent a resonance driving field, whereas those with $m \neq K$ result in the resonance frequency being shifted. In the second order perturbation theory, we obtain

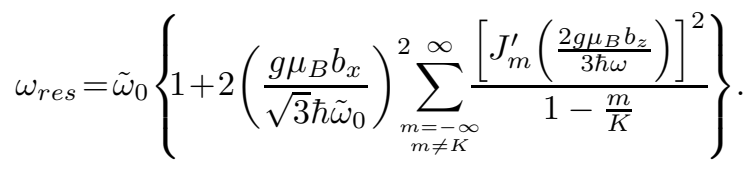


Substitution of $A_{ \pm}$by $A_{ \pm} \exp [\mp i(K \omega t-\pi / 2) / 2]$ leads us to (8) with $\omega_{\text {res }}$ given by (11) and the half width

$$
\Gamma_{K}=\frac{2 g \mu_{B} b_{x}}{\sqrt{3} \hbar}\left|J_{K}^{\prime}\left(\frac{2 g \mu_{B} b_{z}}{3 \hbar \omega}\right)\right| .
$$

We assume that an ion is in the lower hyperfine state when it is injected in a Penning trap at $t=0$. With this initial condition Eqs. (8) can be easily solved for $\lambda \ll \Gamma_{K}$. The probability of finding an ion in the decaying state $|-\rangle$ is given by

$$
\begin{aligned}
& P_{F}(t)=\delta_{F F_{-}}\left[\cosh \left(\frac{\lambda \Delta}{2 \Omega} t\right)-\frac{\Delta}{\Omega} \sinh \left(\frac{\lambda \Delta}{2 \Omega} t\right)\right] e^{-\lambda t / 2}+ \\
& \frac{\left(\delta_{F F_{+}}-\delta_{F F_{-}}\right) \Gamma_{r e s}^{2}}{\Delta^{2}+\Gamma_{r e s}^{2}}\left[\sinh ^{2}\left(\frac{\lambda \Delta}{4 \Omega} t\right)+\sin ^{2}\left(\frac{1}{2} \Omega t\right)\right] e^{-\lambda t / 2},
\end{aligned}
$$

where $\Omega=\sqrt{\Delta^{2}+\Gamma_{K}^{2}}$ is the Rabi frequency and $\Delta=$ $\omega_{\text {res }}-K \omega$ is the detuning. The probability depends on the lower state angular momentum $F=F_{ \pm}$. Expression (13) describes damped oscillations with the frequency $\Omega$ decaying on the scale of half the EC decay constant $\lambda$. Nevertheless, the total number of decays for infinite time calculated with (13) is equal to the number of radioactive ions at $t=0$.

To elucidate the time modulation of EC decay, we utilize the concept of the dressed atom introduced by Shirley [8] as the quantum limit of the semiclassical theory. The ion with $\mu>0$ coasted in a trap and exposed to a resonance field is described by the quantum wave function

$$
\Psi(t)=a_{+}(t)|+\rangle|N-K\rangle+a_{-}(t)|-\rangle|N\rangle,
$$

where $|N\rangle$ and $|N-K\rangle$ are the wave functions with the fixed number of photons having the energy $\hbar \omega$. Thus, the dressed ion has the two hyperfine states entangled with photons. The EC decay at the time $t$ disentangles the state (14) by projecting $\Psi(t)$ on the state $|-\rangle$ with the probability $P_{F_{-}}(t)$ (13), to which the decay rate is proportional.

To observe the time modulation, the $\mathrm{H}$-like radioactive ion has to have the following properties:

(1) Its half-life $T_{1 / 2}$ should be longer than its preparation and cooling time.

(2) It should have a large fraction of the EC decay, implying large $Z$.

(3) The life time $\tau$ of the upper hyperfine state should be longer than $T_{1 / 2}$, implying small $Z$.

(4) The frequency $\Omega$ of the time modulation of the EC decay should be greater than the EC decay constant $\lambda$.

Table II shows H-like ions which may be considered as candidates for the experimental observation of time modulation. The hyperfine splitting $\Delta E$ is calculated by Eq. (11). The mean time $\tau$ for the magnetic dipole transition between the upper and lower hyperfine states is estimated with the expression [12]

$$
\tau=\frac{3 \lambda_{e}}{2 \alpha c}\left(\frac{m c^{2}}{\Delta E}\right)^{3} \frac{2 I+1}{2 F+1},
$$

TABLE I: Hydrogen-like radioactive ions with allowed Gamow-Teller transition $1^{+} \rightarrow 0^{+}$appropriate for observation of the time modulation of the EC decay. The half-lives $T_{1 / 2}$ of nuclear ground state is given for neutral atoms.

\begin{tabular}{ccccccc}
\hline \hline $\begin{array}{c}\text { H-like } \\
\text { ion }\end{array}$ & $\mu$ & $\Delta E$ & $\tau$ & $T_{1 / 2}$ & $\begin{array}{c}Q_{E C} \\
\mathrm{keV}\end{array}$ & $\begin{array}{c}\mathrm{EC} / \beta^{+} \\
\%\end{array}$ \\
\hline${ }^{18} \mathrm{~F}$ & $+0.58^{a}$ & 0.672 & $5.55 \mathrm{y}$ & $109.8 \mathrm{~m}$ & 1655 & $3.3 / 96.7$ \\
${ }^{64} \mathrm{Cu}$ & $-0.217^{b}$ & 8.95 & $10.3 \mathrm{~h}$ & $12.7 \mathrm{~h}$ & 1673 & $43 / 18$ \\
${ }^{68} \mathrm{Ga}$ & $0.0117^{b}$ & 0.596 & $7.95 \mathrm{y}$ & $67.6 \mathrm{~m}$ & 2921 & $8.9 / 88.0$ \\
${ }^{78} \mathrm{Br}$ & $0.13^{b}$ & 9.75 & $15.9 \mathrm{~h}$ & $6.46 \mathrm{~m}$ & 3754 & $8 / 92$ \\
${ }^{82} \mathrm{Rb}$ & $+0.554^{b}$ & 48.0 & $7.20 \mathrm{~m}$ & $1.27 \mathrm{~m}$ & 4378 & $4 / 96$ \\
\hline \hline
\end{tabular}

${ }^{a}$ Calculated with Schmidt's factors $g_{j}^{p}$ and $g_{j}^{n}$.

${ }^{b}$ The data from Ref. [13]. No sign is given, if it is unknown.

where $\lambda_{e}$ is the electron Compton wavelength and $F$ is the angular momentum of the ground state. The H-like ion ${ }^{68} \mathrm{Ga}$ seems to be the best candidate. In Table II we have compared the amplitudes of the two fields with different polarizations calculated from Eqs. (10) and (12) for different photon number $K$ and the fixed width $\Gamma_{K}$. The trap field is $B_{0}=1.0 \mathrm{~T}$. It is seen that the circularly polarized field is more suitable for experimental use.

TABLE II: Strength of the linearly polarized $\left(b_{l}=b_{x}\right.$ for transition (i) with $\omega_{0} / 2 \pi=144 \mathrm{GHz}$ ) and circularly polarized $\left(b_{c}=b_{x}=b_{z}\right.$ for transition (ii) with $\tilde{\omega}_{0} / 2 \pi=126 \mathrm{GHz}$ ) harmonic fields required to observe the $K$-photon resonance with the half width $\Gamma_{K} / 2 \pi=0.1 \mathrm{~Hz}$ in the ion ${ }^{68} \mathrm{Ga}^{30+}$.

\begin{tabular}{cccccc}
\hline \hline$K$ & 1 & 2 & 3 & 4 & 5 \\
\hline$b_{l}, \mathrm{~T}$ & $1.54 \cdot 10^{-11}$ & - & $2.38 \cdot 10^{-3}$ & - & $9.07 \cdot 10^{-2}$ \\
$b_{c}, \mathrm{~T}$ & $1.00 \cdot 10^{-12}$ & $2.62 \cdot 10^{-6}$ & $3.47 \cdot 10^{-4}$ & $3.94 \cdot 10^{-3}$ & $1.69 \cdot 10^{-2}$ \\
\hline \hline
\end{tabular}

Storage ring ESR.-We try now to use multiple transition mechanism for the interpretation of the GSI oscillations. It should be emphasized, however, that this problem is a more complicated one owing to the extremely complex configuration of the ring magnetic field designed to store ions. Moreover, the available experimental information is yet incomplete.

The ESR lattice has periodic structure with the spatial period $L=C / 2$, where $C=108.36 \mathrm{~m}$ is the circumference of the ring. Thus, an ion circulating in the ESR is exposed to the periodic magnetic fields of bending dipole and quadrupole magnets. We consider the two fields, which are in the direction of the $\mathrm{x}$-axis tangent to the main orbit and the z-axis perpendicular to its plane. In the reference frame of a moving ion they can be expressed by the Fourier series

$$
\begin{aligned}
& b_{x}(t)=\sum_{n=1}^{\infty} b_{x n} \sin (2 \pi n \gamma v t / L), \\
& B_{z}(t)=B_{z 0}+\sum_{n=1}^{\infty} b_{z n} \cos (2 \pi n \gamma v t / L),
\end{aligned}
$$


where $t$ is the proper time. For the ions in consideration the Lorentz factor is $\gamma=1.43$. The frequency $\omega_{n}$ of $n$-th harmonic is equal to $n \omega$, where $\omega / 2 \pi=\gamma v / L=5.65$ $\mathrm{MHz}$ is the frequency of the first harmonic and the interval between nearby harmonics. The latter is much greater that the width of the multiphoton resonance at the frequency $\omega_{n}$. Therefore, the resonances with different $n$ do not overlap, and may be considered as independent.

At first we consider the transition, induced by the fields of $n$-th harmonic, between the hyperfine states $F=1 / 2$ and $F=3 / 2$ with the energy $\Delta E \sim 1 \mathrm{eV}$. In principle, the resonance transitions with the Rabi frequency of 0.1 $\mathrm{Hz}$ is possible for very high $n$ and small $K$. However, this is a fallacious result, because the mean life time of the upper $F=3 / 2$ state, $\tau=0,03 \mathrm{~s}$, is much shorter than the modulation period. Hence, the "sterile" state can not be the source of observed oscillations.

Let us next consider the transitions between the Zeeman components of the lower hyperfine state. For the modulation to be possible, the weak interaction operator of the EC decay has to have the different matrix elements for the states with different $M$. We assume that this is the case. Then the two quasi-stationary states exposed to the fields of n-th harmonic evolve according to (2), where $a_{+}$and $a_{-}$are the amplitudes of the lower $|1 / 2,+1 / 2\rangle$ and upper $|1 / 2,-1 / 2\rangle$ states with energies $\epsilon_{1}=\hbar\left(\omega_{+}-i \lambda / 2\right)$ and $\epsilon_{3}=\hbar\left(\omega_{-}-i \lambda / 2\right)$, respectively.

Suppose the ion ${ }^{140} \operatorname{Pr}^{58+}$ (or ${ }^{142} \mathrm{Pm}^{60+}$ ) circulating in the ESR moves along the main orbit. Then it is exposed to the periodic magnetic field of dipole magnets only. One period $L$ contains three identical dipoles arranged symmetrically around the center of the central dipole magnet. The dipole magnet of length $d=6.5 \mathrm{~m}$ involves the strong bending field $\mathbf{B}\left(0,0, B_{0}\right), B_{0}=1,197$ $\mathrm{T}$, and the fringe field $\mathbf{b}\left(b_{x}, b_{u}, b_{z}\right)$. The latter is calculated by the code OPERA [14]. It is localized in the small region $\Delta x=29 \mathrm{~cm}$ on the two sides of each dipole. The component $b_{z}$ decreases monotonically from $B_{0}$ to zero. The component $b_{x}$ has a pulse shaped form with the maximal value $b_{0}=36 \mathrm{mT}$, and the component $b_{y}$ is too small to be taken into account. The Fourier coefficients in (16) are easily found for these components and the matrix elements in (2) are $U_{+}=U_{-}=p f_{n} / n, V_{+-}=$ $V_{-+}=-q g_{n} / n$, where

$$
p=\frac{g \mu_{B} \gamma B_{0}}{\pi}, q=\frac{g \mu_{B} b_{0}}{\pi}, \text { and } \varpi=\frac{g \mu_{B} \gamma B_{0} d}{\hbar L_{0}}
$$

is the Zeeman splitting $\varpi=\omega_{-}-\omega_{+}$. The periodic in $n$ functions $f_{n} \sim g_{n} \lesssim 1$ depend on the lattice parameters. The splitting $\varpi / 2 \pi=5.65 \mathrm{GHz}$ is well above the frequency of the first harmonic. This means that the harmonics with high $n$ are involved in the resonance transitions. Hence, the driving field is weak and we can use the approximate solution of (7). In Table III we show the half width and detuning for the $K$-photon resonance calculated according to the formulas

$$
\Gamma_{K}=\left|\frac{q g_{n}}{\pi \hbar n} J_{K}^{\prime}\left(\frac{2 p f_{n}}{n \hbar \omega_{n}}\right)\right|, \Delta=\varpi-K \omega_{n},
$$

for the harmonics, which are closest to the resonance.

TABLE III: Half width and detuning for the harmonics close to the $K$-photon resonance for the ion ${ }^{140} \mathrm{Pr}^{58+}$ or ${ }^{142} \mathrm{Pm}^{60+}$ moving along the main orbit. Here $K$ is integer value of $\varpi / \omega_{n}$. All frequencies are in the laboratory frame.

\begin{tabular}{cccccccc}
\hline \hline$n$ & $K$ & $\Gamma_{K}(\mathrm{~Hz})$ & $\Delta(\mathrm{MHz})$ & $n$ & $K$ & $\Gamma_{K}(\mathrm{~Hz})$ & $\Delta(\mathrm{MHz})$ \\
\hline 143 & 7 & $1.48 \cdot 10^{-10}$ & -6.92 & 250 & 4 & 24.4 & -2.97 \\
166 & 6 & $2.88 \cdot 10^{-5}$ & 12.9 & 251 & 4 & 0.304 & -18.8 \\
167 & 6 & $5.22 \cdot 10^{-4}$ & -10.9 & 333 & 3 & 18.9 & 0.989 \\
168 & 6 & 0.328 & -34.6 & 500 & 2 & 854 & -2.79 \\
201 & 5 & 0.782 & -22.7 & 999 & 1 & 2420 & 0.989 \\
\hline \hline
\end{tabular}

The large values of $\Delta$ in Table III may be attributed to the inaccurate trajectory being used. In a storage ring, periodic motion of an ion in the axial direction (rotation) is coupled with its periodic motion in the transverse direction (betatron oscillations). The latter leads to an ion being exposed to the magnetic field of quadrupole lenses which change $\Gamma_{K}$ and $\Delta$. However, for the resonance condition $\Delta \sim \Gamma_{K}$ to be realized an ion has to move along a particular, "resonance" orbit. The decays of ions stored on non-resonance orbits generate the background with the conventional exponential law.

The plausible explanation of the observed oscillation amplitude is related to the coupling of the $F=1 / 2$ and $F=3 / 2$ states. The Hamiltonian corresponding to Eqs. (7) offers the coupling due to virtual transitions in the states $\mathrm{F}=1 / 2$ and $3 / 2$ with different $M$ and nonzero photon numbers. The transitions change the energy (like the Lamb shift) and the wave functions of the states $|1 / 2, \pm 1 / 2\rangle$. However, these changes are small due to the weak driving field of the $n$-th harmonic and cannot explain the observed amplitude.

It is not inconceivable also that the coupling of these states is effected by the static or periodic potential $W$ associated with the ESR fields. The solution of the Schrödiger equation for the two hyperfine states gives in the limit $W_{3 / 2, M}^{1 / 2, \pm 1 / 2} / \Delta E \ll 1$ (the matrix elements $W$ is assumed to be real) the new eigenfunctions

$$
\left[\begin{array}{l}
|1\rangle \\
|2\rangle
\end{array}\right]=\left(\begin{array}{cc}
\cos \frac{\theta}{2} & \sin \frac{\theta}{2} \\
-\sin \frac{\theta}{2} & \cos \frac{\theta}{2}
\end{array}\right)\left[\begin{array}{c}
\left|\frac{1}{2}, \frac{1}{2}\right\rangle \\
\left|\frac{1}{2},-\frac{1}{2}\right\rangle
\end{array}\right], \tan \theta=\frac{2(W \cdot W)_{\frac{1}{2},-\frac{1}{2}}^{\frac{1}{2}}}{\hbar \varpi \Delta E} .
$$

With this basis, we find, following the method of [11], the quasienergy function

$$
\Psi(t)=\left(b_{1}|1\rangle+b_{2}|2\rangle e^{-i K \omega_{n} t}\right) e^{-\frac{i}{2}\left(\omega_{-}+\omega_{+}-i \lambda\right) t},
$$

satisfying the initial condition $\Psi(0)=|1\rangle$. Here the coefficients

$$
b_{1}=\cos (\Omega t / 2)-i \frac{\Gamma_{K}}{\Omega} \sin (\Omega t / 2), b_{2}=-i \frac{\Delta}{\Omega} \sin (\Omega t / 2)
$$

represent Rabi oscillations (slow motion). The function (20) describes the evolution of the system and allows to 
calculate the amplitude of the transition of the mother ion from the state $1 s_{1 / 2}, F=1 / 2$ into the daughter nucleus. The probability of the EC decay is proportional to the square of the amplitude averaged over the period of n-th harmonic (fast motion)

$$
P_{E C} \sim|M|^{2}\left[1-\sin \theta+\frac{2 \Gamma_{K}^{2}}{\Omega^{2}} \sin \theta \sin ^{2}\left(\frac{\Omega}{2} t\right)\right] e^{-\lambda t},
$$

where $|M|^{2}=\left|M_{+\frac{1}{2}}\right|^{2}=\left|M_{-\frac{1}{2}}\right|^{2}$ are the squared amplitudes of EC decay calculated with the unperturbed states. Equation (21) describes damped Rabi oscillations with the EC decay constant $\lambda=\lambda_{E C}$ and the amplitude $\sin \theta \sim W^{2} /(\hbar \varpi \Delta E)$. Comparing the latter with observed amplitude 0.2 , we find $W \sim 0,001 \mathrm{eV}$. The electric quadrupole field required to achieve this value is of the order of atomic fields and cannot exist in the ESR. The required magnetic field $\mathbf{B}\left(0, B_{y}, B_{z}\right)$ should be of the order of $20 \mathrm{~T}$, which is greater by a factor 20 than the ESR fields.

Conclusion.-The effect of atomic electron on the EC decay of $\mathrm{H}$-like ions is very important. We have shown for the first time that the EC-decay rates of such ions can be modulated by using the Rabi resonance method with single or multiphoton transitions. For a long time the multiphoton resonance has been subject of intense experimental and theoretical studies in different fields except nuclear physics. We have proposed the experiment with intermediate mass ions stored in a Penning trap which can demonstrate this phenomenon.
In an attempt to understand the GSI oscillations, we have proposed an alternative mechanism involving resonance multiphoton transitions, induced by the periodic magnetic field of the ESR, between the magnetic substates of the ground $F=1 / 2$ state of the radioactive ions ${ }^{140} \mathrm{Pr}^{58+}$ and ${ }^{142} \mathrm{Pm}^{60+}$. The mechanism includes the coupling of $F=1 / 2$ and $F=3 / 2$ hyperfine states to explain the oscillation amplitude. The important point of this scenario is that the predicted EC decay constant does not violate experimentally and theoretically established ratio $\lambda_{E C}^{H-\text { like }} / \lambda_{E C}^{H e-l i k e} \approx 3 / 2[15]$. We find that the periodic magnetic field of the ESR can generate the oscillations with the frequency of about $0.1 \mathrm{~Hz}$ in the transitions involving several photons. However, the field required to explain the observed amplitude should be many times higher than that of the ESR. Moreover, to tune to the resonance a stored ion should move along specific, "resonance" orbits. This cast some doubts upon the involvement of the hyperfine structure in the observed oscillations. Therefore, it is necessary to perform the single-ion experiment with the ions ${ }^{123,5} \mathrm{Cs}^{54+}$ having the ground state $1 s_{1 / 2} F=0$ or with the He-like ions without a hyperfine structure. From these experiments definite conclusions may be drawn regarding competing hypotheses for the GSI oscillations.

Acknowledgments.- The author is grateful to A. Dolinski for helpful information concerning storage ring physics, and to A. L. Barabanov and S. T. Belyaev for critical reading of the manuscript and useful comments. The work was supported by the Grant NS-3004.2008.2.
[1] E. Segré, Phys. Rev. 71, 274 (1947).

[2] Y. Litvinov et al.., Phys. Lett. B 664, 162 (2008).

[3] A. N. Ivanov and P. Kienle, Phys. Rev. Lett. 103, 062502 (2009).

[4] V. M. Shabaev et al., Phys. Rev. A 37, 4685 (1988).

[5] L. S. Brown and G. Gabrielse, Rev. Mod. Phys. 58, 233 (1986).

[6] Yu. Novikov, private communication (2009).

[7] L. D. Landau and E. M. Lifshitz // Quantum Mechanics (Pergamon, Oxford, 1958).

[8] J. H. Shirley, Phys. Rev. 138, B979 (1965).
[9] D. F. Zaretskii and V. P. Krainov, Zh. Eksp. Teor. Fiz. 66, 537 (1974) [Sov. Phys. JETP. 39, 257 (1974)].

[10] R. E. Duvall, E. J. Valeo, and C.R. Oberman, Phys. Rev. A 37, 4685 (1988).

[11] H. Sambe, Phys. Rev. A 7, 2203 (1973).

[12] I. I. Sobelman// Atomic Spectra and Radiative Transitions (Springer-Verlag, Berlin/New York, 1979).

[13] N. J. Stone, At. Data Nucl. Data Tables 90, 75 (2005).

[14] A. Dolinski, private communication (2009).

[15] N. Winckler et al., arXiv:0907.227\%v1[nucl-th]. 